PAPER

\title{
Anticipation and phenotype in familial intracranial aneurysms
}

\author{
Y M Ruigrok, G J E Rinkel, C Wijmenga, J van Gijn
}

J Neurol Neurosurg Psychiatry 2004;75:1436-1442. doi: 10.1136/jnnp.2003.025098

See end of article for authors' affiliations

.....................

Correspondence to: Dr Y M Ruigrok,

Department of Neurology,

Rudolf Magnus Institute of

Neuroscience, University

Medical Centre Utrecht

PO box 85500,3500 GA

Utrecht, Netherlands;

ij.m.ruigrok@neuro.azu.nl

Received 7 August 2003

Revised 4 December 2003

Accepted 14 January 2004
Background: In familial intracranial aneurysms there is evidence for genetic heterogeneity, probably from mutations at separate loci.

Objectives: To compare demographic and clinical features in patients of families with familial intracranial aneurysm and different patterns of inheritance; and to compare the ages of patients with subarachnoid haemorrhage (SAH) in affected parent-child pairs to determine whether there is anticipation.

Methods: Pedigrees for 53 families with familial intracranial aneurysms were constructed, divided into patterns of inheritance suggestive or not suggestive of autosomal dominant transmission. Demographic and clinical features were compared. The age at time of SAH in affected parent-child pairs was compared using the Wilcoxon test.

Results: No differences in demographic or clinical features were found between families compatible with an autosomal dominant pattern of inheritance and those with a non-dominant pattern. In families with affected members in two successive generations the age at time of SAH in parents was 55.2 years and in children 35.4 years (mean difference, 19.8 years, $\mathrm{p}<0.001$ ).

Conclusions: Phenotypes are similar in families with and without a probable autosomal dominant pattern of inheritance. Thus in future genetic studies on familial intracranial aneurysms, stratification according to phenotype is not likely to be useful. Anticipation probably occurs, as affected parents are significantly older at the time of SAH than their affected children.
$\mathrm{F}$ amilial intracranial aneurysms are defined by the presence of at least two affected first degree relatives with aneurysmal subarachnoid haemorrhage (SAH) or an unruptured intracranial aneurysm. In comparison with sporadic intracranial aneurysms, familial aneurysms rupture at an earlier age and are more often located at the middle cerebral artery. ${ }^{1-3}$

Some pedigrees of familial intracranial aneurysms seem consistent with an autosomal dominant pattern of inheritance, whereas others suggest a multifactorial or autosomal recessive pattern. Segregation analysis has shown that several mendelian inheritance modes were compatible, but that autosomal dominant and autosomal recessive transmission were the most likely patterns. ${ }^{4}$ Thus genetic heterogeneity in familial intracranial aneurysm is possible, either with mutations at separate loci (locus heterogeneity) or with different mutations at the same locus (allelic heterogeneity). Recently, evidence for possible locus heterogeneity in familial intracranial aneurysms was found as one study reported that a genome-wide scan for intracranial aneurysm susceptibility genes showed positive evidence for linkage on $7 q 11,{ }^{5}$ while another found linkage on 19q. ${ }^{6}$ Locus heterogeneity may be characterised by differences in phenotype.

An example of a genetically heterogeneous disorder is Alzheimer's disease. In genetic studies on Alzheimer's disease the patients are dichotomised according to phenotype into those with early onset and those with late onset, and different genetic deficits have been identified in the two distinct subgroups. ${ }^{7}$ In case there is a difference in phenotype in familial intracranial aneurysms, future genetic studies on this condition might also use stratification according to phenotype to optimise mutational screening.

We compared demographic and clinical features between patients of families consistent with an autosomal dominant pattern of inheritance with those where the pattern of inheritance was not suggestive of autosomal dominant transmission (non-dominant mode). In addition, in families with an autosomal dominant transmission we compared the ages at the time of SAH in parent-child pairs, because a previous study indicated that in two successively affected generations the age at time of SAH was lower in children than in their parents, which suggests genetic anticipation. ${ }^{1}$

\section{METHODS}

\section{Ascertainment of families and definition of familial} intracranial aneurysms

For this study we had intended to define familial intracranial aneurysms as the presence of at least two first degree relatives with aneurysmal $\mathrm{SAH}$, in contrast to the commonly used definition (the presence of at least two affected first degree relatives with aneurysmal SAH or an unruptured intracranial aneurysm), as the latter will result in the inclusion of families where only one relative had SAH and one other had an unruptured intracranial aneurysm. Inclusion of such families is likely to lead to bias because many have been ascertained differently from those where two members have had an SAH (for example, by active screening of asymptomatic relatives of an SAH patient). However, because most other genetic studies on familial intracranial aneurysms have included families with one SAH and one unruptured aneurysm, we carried out two separate analyses using both definitions: (1) defined as the presence of at least two affected first degree relatives with aneurysmal SAH; (2) defined as the presence of at least two affected first degree relatives with aneurysmal SAH or with an unruptured intracranial aneurysm.

We used records of patients with familial intracranial aneurysms known in the University Medical Centre Utrecht from two previous studies, collected between September 1991 and October $1992^{1}$ and from December 1995 to March 1997. ${ }^{8}$ In the first study patients with at least two first degree relatives with SAH were selected from a prospectively 
collected series of patients with aneurysmal SAH. ${ }^{1}$ The second study used magnetic resonance (MR) angiography to screen for intracranial aneurysms in first degree relatives of a consecutive series of index patients with aneurysmal SAH. ${ }^{8}$ We also included families from the outpatient clinic for intracranial aneurysms of the University Medical Centre Utrecht. Members of these families visited the outpatient clinic at their own request or after referral by a neurologist or a neurosurgeon. All medical documents on family members with a medical history suggestive of SAH, intracerebral haemorrhage, stroke, or unruptured intracranial aneurysm were reviewed. Asymptomatic individuals were considered eligible for screening if they were related in the first degree to at least two patients with SAH or an unruptured intracranial aneurysm. These persons were screened with MR angiography. First degree relatives who chose not to visit the outpatient clinic were not actively invited to be screened.

\section{Inclusion and exclusion criteria}

Index patients with aneurysmal SAH were defined by symptoms suggestive of SAH combined with subarachnoid blood on computed tomography (CT) and a proven aneurysm on CT angiography or conventional angiography. In patients who died before angiography could be done, the pattern of haemorrhage on CT had to compatible with a ruptured aneurysm. Patients with an unruptured intracranial aneurysm were identified by CT or MR angiography, conventional angiography, surgery, or necropsy. Episodes of SAH in relatives were categorised into "definite" or "probable" $\mathrm{SAH}$. The diagnosis of definite SAH was based on clinical features suggestive of SAH in combination with either subarachnoid blood (as demonstrated by CT or analysis of the cerebrospinal fluid), or an intracranial aneurysm proved by angiography (conventional angiogram, CT angiogram, or MR angiogram), surgery, or necropsy. Probable SAH was defined as either sudden severe headache in combination with a normal neurological examination, and haemorrhagic CSF followed by sudden deterioration and death within four weeks (consistent with rebleeding), or as a history describing a second ictus followed by death within the first four weeks after "stroke" in a person aged less than 70 years.

We constructed pedigrees for each family and determined the mode of inheritance. We defined an autosomal dominant pattern of inheritance as the presence of at least two affected relatives (with SAH or an unruptured intracranial aneurysm) in two successive generations, or at least two affected half brothers or half sisters. If only siblings were affected and if one of the parents had died before 60 years of age, while the cause of death was other than SAH or unknown, the parent was considered as being "non-informative" and the family was excluded from the analysis. If only siblings were affected and both parents were still alive or had reached an age above 60 years without having had an SAH, families were defined as having a pattern of inheritance suggestive of a mode of inheritance other than autosomal dominant. These families will be further referred to as having a non-dominant pattern of inheritance. Families with autosomal dominant polycystic kidney disease (ADPKD) and connective tissue disorders such as Ehlers-Danlos disease were excluded.

\section{Data collected}

For the SAH patients, we collected data on age at time of $\mathrm{SAH}, \mathrm{sex}$, number and location of ruptured and unruptured intracranial aneurysms, and outcome after SAH (on discharge from the hospital). For outcome on discharge from the hospital we used the modified Glasgow outcome scale (GOS) with three different categories: independent (GOS 4 and 5), dependent (GOS 2 and 3), or dead (GOS 1). ' Where a patient had a second SAH later in life, we used only the data on the first SAH. In patients with an unruptured intracranial aneurysm we only studied sex and the number and location of any intracranial aneurysms.

\section{Literature search}

To compare our data on age, sex, and number and location of ruptured and unruptured intracranial aneurysms with those in earlier studies, we carried out a Medline search for articles in English on familial intracranial aneurysms from 1954 to 2002 using the key words aneurysm, cerebral, intracranial, subarachnoid haemorrhage, genetics, and familial in different combinations. We also scrutinised the reference lists of all publications retrieved for additional studies. In these families we applied only the strictest definition of familial intracranial aneurysms (the presence of at least two first degree relatives with aneurysmal SAH; see paragraph on ascertainment of families above). We used the same inclusion and exclusion criteria as for our own families with familial intracranial aneurysm, as described above. In addition, we included only families with complete information on age at time of SAH, sex, and number and location of ruptured intracranial aneurysms for all affected subjects. We excluded reports of families with only affected siblings and no information on the parents. Twin studies were excluded.

\section{Data analyses}

As described in the paragraph on ascertainment of families, we carried out two separate analyses using the two different definitions of familial intracranial aneurysm. The demographic and clinical features were compared in patients from families with an autosomal dominant pattern of inheritance and with a non-dominant pattern. Age at the time of SAH was compared in the two groups by calculating the difference in mean age with the corresponding 95\% confidence interval (CI). This analysis may be influenced by ascertainment bias, as individuals with early onset disease (that is, SAH at a young age) may be referred early to a specialist and be diagnosed as having familial intracranial aneurysm, whereas patients with late onset disease may not come to medical attention. To correct for this possible bias we also conducted this analysis excluding all parent-child pairs involving a proband. For the remaining features we assessed the proportions of the characteristics and calculated the differences between these proportions with corresponding 95\% confidence intervals. In the families with an autosomal dominant pattern of inheritance and two successive generations of patients with $\mathrm{SAH}$, the ages at onset of SAH in the different generations were compared using the Wilcoxon test for non-parametric comparison of paired samples. Analysis of variance (ANOVA) was used to test whether the distribution of the difference between ages at onset in parent-child pairs differed according to the sex of the affected parent.

\section{RESULTS}

Using the strict definition of familial intracranial aneurysms (presence of at least two first degree relatives with aneurysmal SAH), we included 36 families. Of these, 17 had an autosomal dominant pattern of inheritance and 19 a non-dominant pattern (fig $\mathrm{IA}$ and $\mathrm{IB}$ ). In these 36 families, 84 members had had an SAH and 11 had been treated for unruptured aneurysms. In all but one of the 17 families with an autosomal dominant inheritance the conclusion was based on subsequent generations being affected, with $41 \%$ (95\% CI, $28 \%$ to $55 \%$ ) of the siblings in the second generation affected. In the remaining family, half brothers or half sisters were affected. In the 19 families with non-dominant pattern of inheritance, $34 \%$ (95\% CI, $26 \%$ to $42 \%$ ) of siblings were affected. In one of these families the parents were 
A
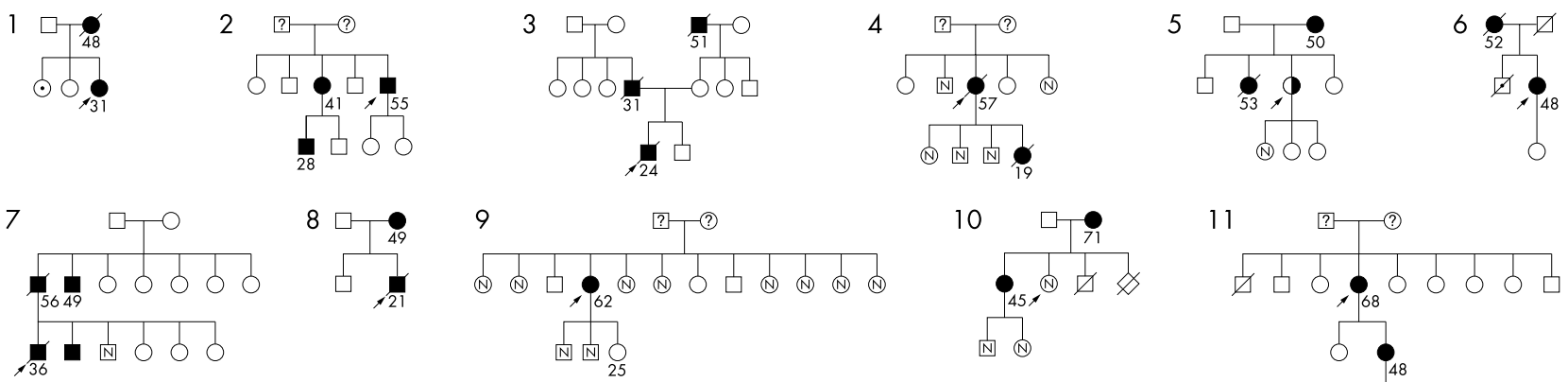
10

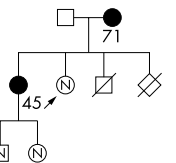
$\mathbb{N}$
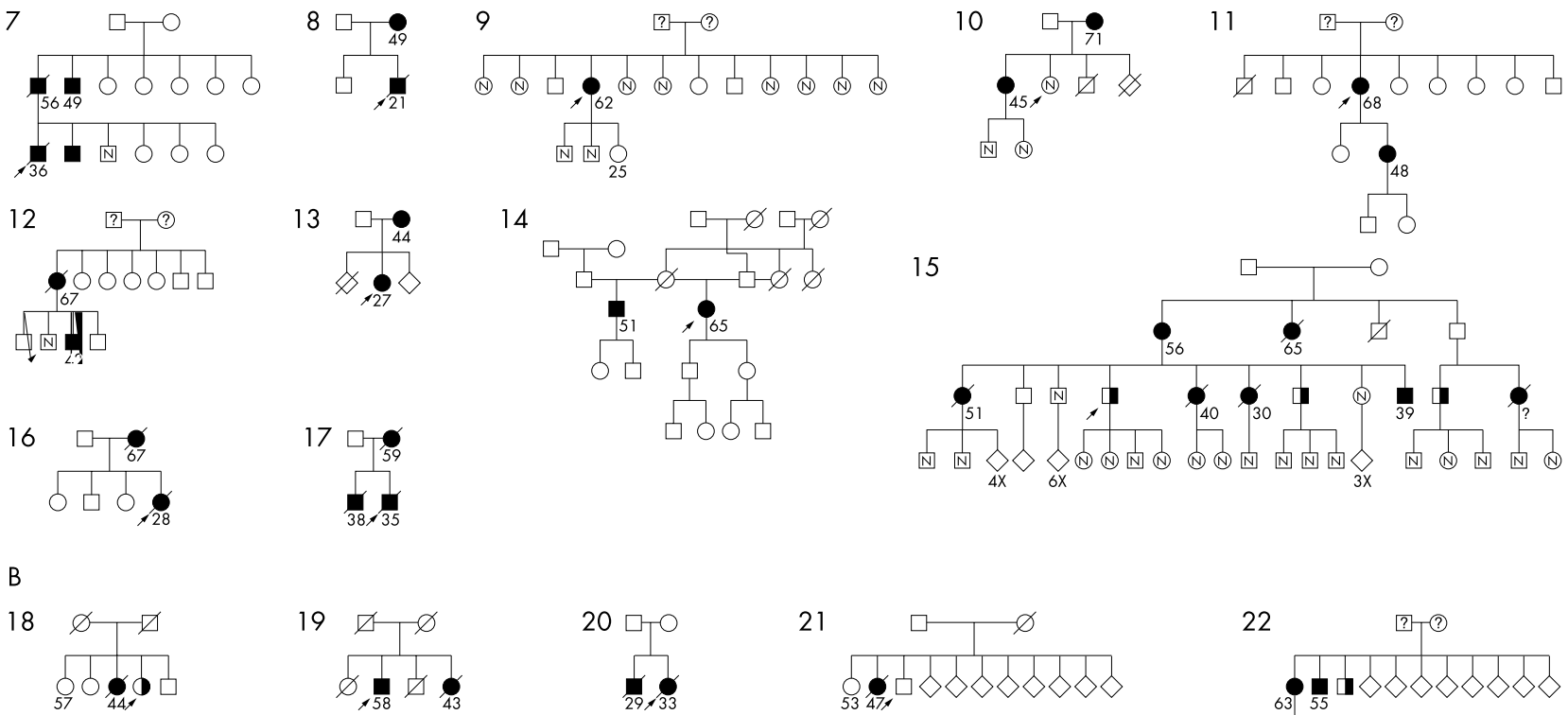

B

18

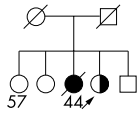

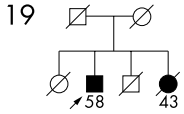

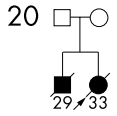

24

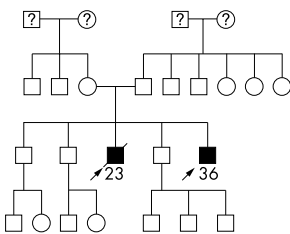

21
23

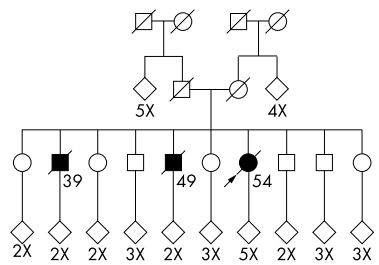

27
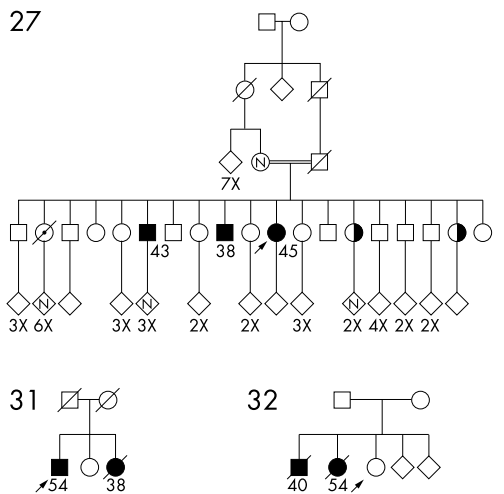

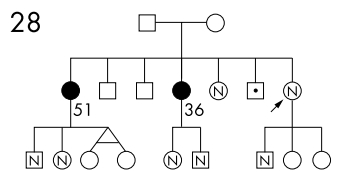

29
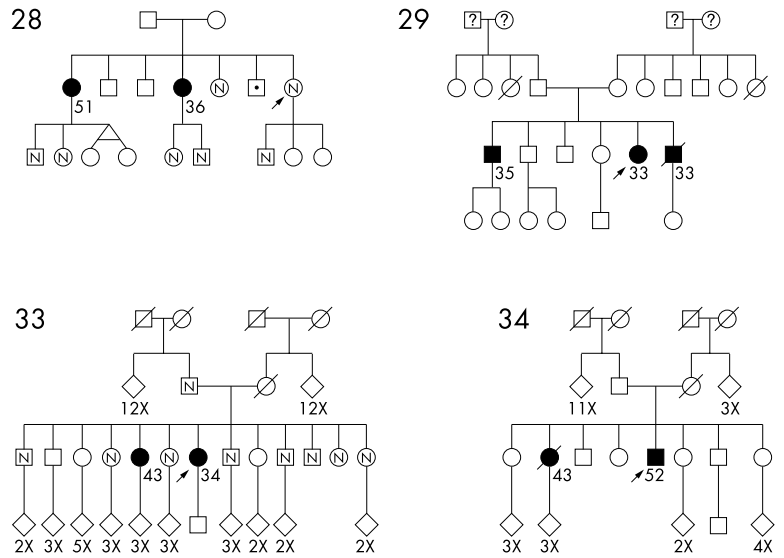

25

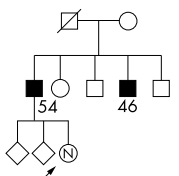

22

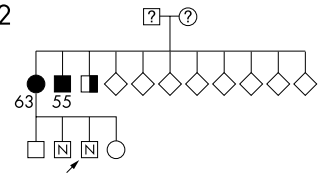

26

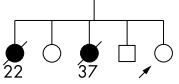

36

$\nabla \quad \varnothing$
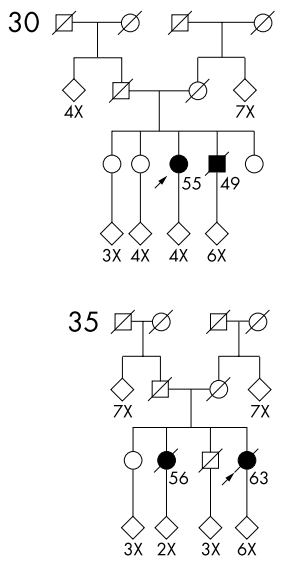

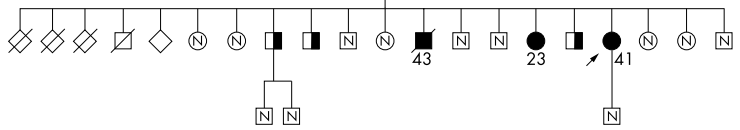

\begin{tabular}{|llllll|}
\hline$\bigcirc$ & Women & $\square_{54}$ & SAH, with age of SAH & $\square$ & Data unknown \\
$\square$ & Men & $\square$ & Unruptured IA & $\Rightarrow$ & Proband \\
$\diamond$ & Sex unknown & $\mathbb{N}$ & Angiography negative & $\diamond_{4 \mathrm{X}}$ & Number of relatives \\
$\varnothing$ & Deceased & $\square$ & Possible SAH & & \\
\hline
\end{tabular}

Figure 1 (A) Families with familial intracranial aneurysms with an autosomal dominant pattern of inheritance, defined as the presence of at least two affected first degree relatives with aneurysmal subarachnoid haemorrhage (SAH). (B) Families with familial intracranial aneurysms with a nonautosomal dominant pattern of inheritance, defined as the presence of at least two affected first degree relatives with aneurysmal SAH. 
consanguineous (first cousins), which suggests an autosomal recessive inheritance (fig $1 \mathrm{~B}$, family 27 ).

When we used the wider definition of familial intracranial aneurysms (presence of at least two affected first degree relatives with aneurysmal $\mathrm{SAH}$ or with an unruptured intracranial aneurysm) we were able to include 17 additional families: eight with an autosomal dominant pattern of inheritance and nine with a non-dominant pattern (fig $2 \mathrm{~A}$ and 2B). In 53 families ( 36 plus 17), 102 members had had an SAH and 36 had been treated for unruptured aneurysms. Of the 25 families with autosomal dominant inheritance, 22 had two or three affected generations, with 44\% (95\% CI, $33 \%$ to $55 \%$ ) of the siblings of the second or third generation affected. In the other three families, half brothers or half sisters were affected. In the 28 families with a non-dominant pattern of inheritance $33 \%$ (95\% CI, $26 \%$ to $39 \%$ ) of siblings were affected.

In two of the 25 families with an autosomal dominant pattern of inheritance three successive generations of patients had intracranial aneurysms (fig 2A: families 38 and 39). In family 39, patient III-1 had an SAH from an intracranial aneurysm of the middle cerebral artery, while screening with MRA did not show an intracranial aneurysm in her mother. The most likely explanation is a reduced penetrance or anticipation, as the mother may still develop an intracranial aneurysm in the future.

In our literature search for families with familial intracranial aneurysm we identified 34 families. ${ }^{10-32}$

\section{Demographic and clinical features}

In table $\mathrm{l}$ we summarise the demographic and clinical features for patients from families with at least two first degree relatives with aneurysmal SAH (our preferred definition of familial intracranial aneurysm) separately for our own observations and those in previous studies. We found no difference in mean age at the time of SAH between patients with autosomal dominant and non-dominant patterns of inheritance. Also, the proportion of women or multiple intracranial aneurysms did not differ between the two groups, and found no differences in the location of the intracranial aneurysms. The outcome after SAH was similar in patients with a dominant and a non-dominant pattern of

A

37
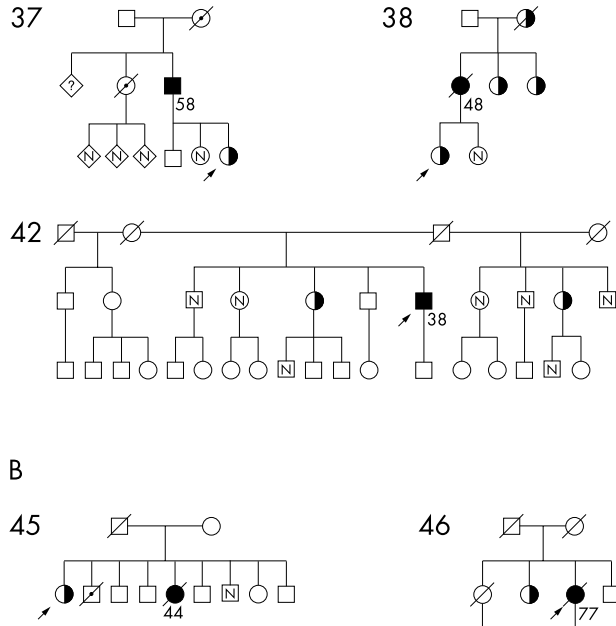

50

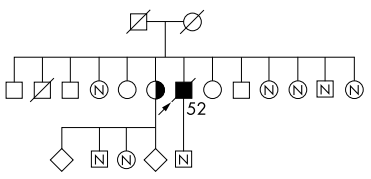

38

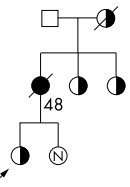

46

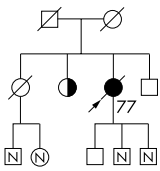

39

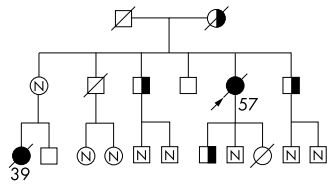

43

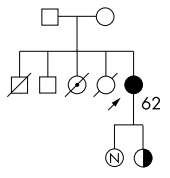

40

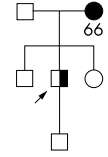

44

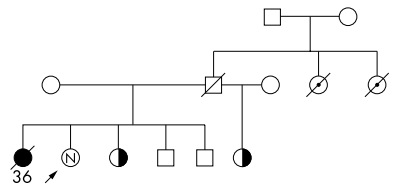

47

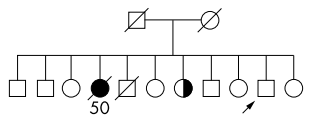

52

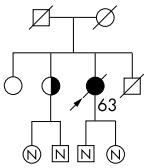

48

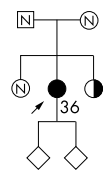

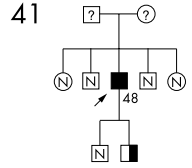

田

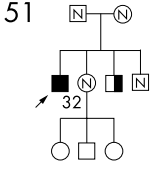

$\square \quad$ Deceased

$\boldsymbol{\square}_{54} \mathrm{SAH}$, with age of SAH

- Unruptured IA
$\mathbb{N}$ Angiography negative

$\odot$ Possible SAH

2] Data unknown

Figure 2 (A) Additional families with an autosomal dominant pattern of inheritance. Family 38: At the age of 48, patient II: 1 collapsed and computed tomography (CT) showed a subarachnoid haemorrhage with an intracerebral haematoma suggestive of an aneurysm of the left middle cerebral artery. She died before angiography could be done. Screening with conventional angiography showed an aneurysm of the anterior communicating artery in both her sisters (patients II:2 and II:3) and an aneurysm of the right middle cerebral artery in her mother (patient I:2). Several years later patients III: 1 and III: 2 were screened for intracranial aneurysms with magnetic resonance angiography (MRA). In patient III:1 an aneurysm of the top of the basilar artery was found. Family 39: Patient II:5 was 59 years of age when she suddenly collapsed. CT showed a subarachnoid haemorrhage. She died before angiography could be done. Her daughter (patient III:9) was diagnosed with an arteriovenous malformation from the vena magna Galeni, from which she had recurrent intracerebral haemorrhages and died at the age of 24. Screening with MRA showed an aneurysm of the anterior communicating artery in patient II-3, of the left middle cerebral artery in patient II:6, and of the right middle cerebral artery in patient III:7. Patient III: 1 at age 39 years was found unconscious approximately three years after screening of her family members. CT showed subarachnoid blood; CT angiography demonstrated an aneurysm of the left middle cerebral artery. Four years after screening patient I:2 died at age 94 . Necropsy revealed an unruptured aneurysm of the anterior communicating artery. (B) Additional families with a non-autosomal dominant pattern of inheritance. 
Table 1 Comparison of characteristics of patients with familial intracranial aneurysms (defined as the presence of at least two first degree relatives with aneurysmal subarachnoid haemorrhage) and an autosomal dominant or non-dominant pattern of inheritance (families from the present study and from published reports)

\begin{tabular}{|c|c|c|c|c|c|c|}
\hline & \multicolumn{3}{|l|}{ This study } & \multicolumn{3}{|c|}{ Published reports } \\
\hline & $A D(n)$ & Non-AD (n) & Difference $(95 \% \mathrm{Cl})$ & $A D(n)$ & Non-AD (n) & Difference $(95 \% \mathrm{Cl})$ \\
\hline $\begin{array}{l}\text { Mean age at time of SAH } \\
\text { (including proband) (years) }\end{array}$ & $\begin{array}{l}45.0(42) \text {, } \\
\text { range } 21 \text { to } 71\end{array}$ & $\begin{array}{l}43.7(42), \\
\text { range } 22 \text { to } 63\end{array}$ & $1.3(-4.5$ to 6.7$)$ & 44.9 (49) & $49.5(29)$ & $4.6(-2.2$ to 11.0$)$ \\
\hline $\begin{array}{l}\text { Mean age at time of SAH } \\
\text { (excluding proband) (years) }\end{array}$ & $\begin{array}{l}46.7(29) \text {, } \\
\text { range } 24 \text { to } 71\end{array}$ & $\begin{array}{l}42.5(31) \text {, } \\
\text { range } 22 \text { to } 64\end{array}$ & $4.2(-1.8$ to 10.0$)$ & & & \\
\hline Proportion of women & $63 \%(29)$ & $55 \%(27)$ & $8(-12$ to 28$)$ & $65 \%(33)$ & $62 \%(21)$ & $3(-18$ to 24$)$ \\
\hline Multiple intracranial aneurysms & $18 \%(6)$ & $26 \%(9)$ & $-8(-27$ to 11$)$ & $11 \%(6)$ & $14 \%(5)$ & $-3(-17$ to 11$)$ \\
\hline \multicolumn{7}{|l|}{ Outcome } \\
\hline Dead & $43 \%(18)$ & $43 \%(17)$ & $0(-21$ to 22$)$ & & & \\
\hline Dependent & $5 \%(2)$ & $10 \%(4)$ & $-5(-17$ to 6$)$ & & & \\
\hline Independent & $52 \%(22)$ & $48 \%(19)$ & $4(-17$ to 27$)$ & & & \\
\hline \multicolumn{7}{|l|}{ Site of intracranial aneurysms } \\
\hline ACA & $48 \%(19)$ & $35 \%(17)$ & $13(-8$ to 33$)$ & $25 \%(14)$ & $21 \%(8)$ & $4(-13$ to 21$)$ \\
\hline MCA & $25 \%(10)$ & $29 \%(14)$ & $-4(-22$ to 15$)$ & $21 \%(12)$ & $31 \%(12)$ & $-10(-28$ to 8$)$ \\
\hline ICA & $23 \%(9)$ & $29 \%(14)$ & $-6(-24$ to 12$)$ & $48 \%(27)$ & $49 \%(18)$ & $-1(-21$ to 19$)$ \\
\hline VBA & $5 \%(2)$ & $8 \%(4)$ & $-3(-13$ to 7$)$ & $7 \%(4)$ & $3 \%(1)$ & $4(-4$ to 13$)$ \\
\hline
\end{tabular}

$A C A$, anterior communication artery complex; $A D$, autosomal dominant inheritance; non- $A D$, mode of inheritance other than autosomal dominant; $C l$, confidence interval; ICA, internal carotid artery complex (including posterior communicating artery); MCA, middle cerebral artery complex; SAH, subarachnoid haemorrhage; VBA, vertebrobasilar artery complex.

inheritance. When we used the wider definition of familial intracranial aneurysms, we again found no difference between families with a dominant and and a non-dominant pattern of inheritance according to age at time of SAH, sex, and proportion of patients with multiple intracranial aneurysms (data not shown). Also, we did not find differences in the location of ruptured and unruptured intracranial aneurysms in these families.

\section{Tests for anticipation effect}

In the families with an autosomal dominant pattern of inheritance and SAH in two successive generations, the age of the child at the time of the haemorrhage was lower than the age of the parent in 19 of the 20 pairs (fig lA). Seven of these pairs have been reported before. ${ }^{1}$ The mean age of the parent at the time of the SAH was 55.2 years and that of the child, 35.4 years (difference 19.8 years, range -3 to 38 ). The difference in mean age between the two generations was statistically significant $(\mathrm{p}<0.001)$. After exclusion of all parent-child pairs involving a proband from each pedigree, the mean age of the parent at the time of the SAH was 56.8 years and that of the child, 40.2 years (difference 16.6 years, range -3 to 26 years). This difference in mean age between the two generations was again statistically significant $(p=0.007)$. In all families from the literature with SAH in two successive generations (27 parent-child pairs, of which 21 have been reviewed before $^{1}$ ), the age at time of SAH was lower in the child. In these families from previous reports, the mean age of the parent at the time of the SAH was 56.6 years against 34.6 years for the child (difference 22.0 years); this difference was statistically significant $(\mathrm{p}<0.001)$.

The mean difference in age at onset between parent and child was 20.5 years if the parent was a mother $(n=18)$ and 13.5 years if the parent was a father $(n=2)$. This difference was not statistically significant $(p=0.28)$. In the families reported previously, this mean difference was 21.1 years if the parent was a mother $(n=22)$ and 26.0 years if the parent was a father $(\mathrm{n}=5)(\mathrm{p}=0.38)$.

\section{DISCUSSION}

In this study we report a large number of families with familial intracranial aneurysms. We found no differences in demographic or clinical features between affected members from families with a pattern of inheritance suggestive of autosomal dominant transmission and those from families with a pattern of inheritance not suggestive of autosomal dominant transmission (non-dominant mode). Our results imply that stratification according to phenotype is not possible in future genetic studies on familial intracranial aneurysms. Of course, absence of differences in phenotype between patients with an autosomal dominant pattern and patients with a seemingly non-dominant pattern does not preclude locus heterogeneity.

We extended upon a previous observation, based on smaller numbers, that the mean age of parents at the time of SAH is significantly higher than that of their affected children. ${ }^{1}$ This phenomenon was consistent throughout 19 of the 20 families, and corroborates the notion of clinical anticipation. In these analyses the number of male parents was much smaller than the number of affected mothers. We therefore could not demonstrate an effect of the sex of the affected parent on anticipation and childhood onset of intracranial aneurysm. Comparing outcome, number, and size of aneurysms in parent-child pairs probably will not further substantiate the existence of anticipation, as the association between multiple aneurysms and poor outcome has not been demonstrated, ${ }^{33}$ and large aneurysms are only associated with a small increase in risk of poor outcome. ${ }^{34}$ It is not possible to demonstrate such a small increase in our limited study population of 20 parent-child pairs.

A shortcoming of our study is that no systematic screening of all first degree family members was undertaken, which may have led to some bias. Because screening was incomplete, some families may only show an autosomal dominant pattern of inheritance later on, as the parents or the children of the affected sibs may harbour undetected intracranial aneurysms. It is also possible that these parents and children will develop intracranial aneurysms in the future. Theoretically, inclusion of families in the group with a non-dominant mode that later appear to have a dominant mode of inheritance may have obscured true differences.

Anticipation can be assumed erroneously, as a result of several kinds of ascertainment bias. As early as 1948 Penrose mentioned three sources of error. ${ }^{35}$ The first is selection of parents with late onset by limitation of reproduction in early onset patients. This form of bias might operate in familial intracranial aneurysm. The mean age of SAH in familial (and sporadic) intracranial aneurysm is higher than the reproductive age, but $22 \%$ of the female $\mathrm{SAH}$ patients are still younger than 45 years of age. ${ }^{36}$ 
The second potential source of bias is the early diagnosis of severe early onset disease and the late recognition of milder late onset disease. As we analyse only the onset of SAH and as the onset of SAH is sudden and requires prompt medical attention regardless of family history, this source of bias does not seem to play a role here. Furthermore, we attempted to adjust for this tendency by undertaking a separate analysis with exclusion of all parent-child pairs involving an index patient.

The third source of bias is that of index selection, caused by the problem that pairs consisting of a parent with early onset disease and a child with late onset disease are unlikely to be ascertained by a study, given the large span of time separating the two events. To adjust for this type of bias, the optimal design study should be carried out in a stable study population with a high yield of case ascertainment over a long period of time. Unfortunately, it is almost impossible to conduct such a study, as it would last several decades. Another solution might be to consider only the families where the last generation children were born a long time ago, for example before 1920. However, with such an analysis no patients at all would be left in our study or in any other.

For our study we excluded six parent-child pairs because the episode suggestive of SAH could not be proven with certainty in the child or the parent. In five of these the parent was older than the child at the time of the episode suggestive of SAH. The differences in age in these excluded parent-child pairs were comparable to those of the parent-child pairs included in our study. Based on these results, the bias of index selection is probably small in our study.

Clinical anticipation may be explained by the transmission of an unstable trinucleotide repeat sequence that increases in size down successive generations. The seven autosomal dominant disorders so far described with unstable mutations (myotonic dystrophy, Huntington disease, spinocerebellar ataxia types 1, 2, 3, and 7, and dentatorubral pallidolysian atrophy) all show anticipation. ${ }^{37}{ }^{38}$ On the other hand, clinical anticipation may be explained by an increased exposure to risk factors for aneurysmal SAH-such as smoking, alcohol consumption, and hypertension ${ }^{39}$-in affected children compared with their affected parents. Further studies are needed to unravel the cause of the notion of clinical anticipation.

We observed a single family with a pattern suggestive of an autosomal dominant mode of inheritance and an unaffected parent-that is, no aneurysm on MR angiography-with affected offspring. As anticipation is likely to be involved in the genetics of familial intracranial aneurysm, the unaffected parent may still develop an aneurysm in the future. Alternatively, this phenomenon may indicate reduced penetrance.

The families with a pattern of inheritance suggestive of a non-dominant transmission may represent a heterogeneous group. For example, some of these families may turn out to have an autosomal dominant pattern of inheritance if the parents or the children of the patients develop intracranial aneurysms later on. In other families genetic factors may play a minor role in that siblings could have been affected by chance and not so much by genetic factors, given the approximately $2 \%$ rate of unruptured intracranial aneurysms in the general population. ${ }^{40}$ Also families with polygenetic inheritance may have been included. Furthermore, at least one of the families with a non-dominant transmission may have a transmission mode compatible with an autosomal recessive pattern as the parents of the affected sibs were consanguineous.

Two families with an autosomal dominant pattern of inheritance showed intracranial aneurysms in three successive generations. Such families are rare, probably because $\mathrm{SAH}$ and intracranial aneurysms could be diagnosed with certainty only in the past few decades, following the introduction of catheter angiography and CT. Shinton et al ${ }^{41}$ reported a family with patients with SAH in three successive generations, but aneurysms were shown in only two generations. In a family described by Schievink et al, a patient of the third generation died from an episode suggestive of SAH, but the diagnosis could not be confirmed as necropsy was not carried out. ${ }^{4}$

\section{Conclusions}

In familial intracranial aneurysm, phenotypes are similar in families with probable autosomal dominant and nondominant patterns of inheritance. There is no indication that in future genetic studies on familial intracranial aneurysms stratification according to phenotype can be used. Anticipation is very probable in familial intracranial aneurysm.

\section{ACKNOWLEDGEMENTS}

This work was in part supported by an established clinical investigator grant from the Netherlands Heart Foundation to GJER (grant D98.014). YMR is supported by the Netherlands Organisation for Scientific Research (NWO), project No 940-37-023.

\section{Authors' affiliations}

Y M Ruigrok, G J E Rinkel, J van Gijn, Department of Neurology, Rudolf Magnus Institute of Neuroscience, University Medical Centre Utrecht, Utrecht, Netherlands

C Wijmenga, Department of Biomedical Genetics, University Medical Centre Utrecht

Competing interests: none declared

\section{REFERENCES}

1 Bromberg JEC, Rinkel GJE, Algra A, et al. Familial subarachnoid hemorrhage: distinctive features and patterns of inheritance. Ann Neurol 1995; 38:929-34.

2 Lozano AM, Leblanc R. Familial intracranial aneurysms. J Neurosurg 1987;66:522-8.

3 Norrgard O, Angquist KA, Fodstad $\mathrm{H}$, et al. Intracranial aneurysms and heredity. Neurosurgery 1987;20:236-9.

4 Schievink WI, Schaid DJ, Rogers HM, et al. On the inheritance of intracranial aneurysms. Stroke 1994;25:2028-37.

5 Onda H, Kasuya H, Yoneyama T, et al. Genomewide-linkage and haplotypeassociation studies map intracranial aneurysm to chromosome $7 q 11$. Am J Hum Genet 2001:69:804-19.

6 Olson JM, Vongpunsawad S, Kuivaniemi H, et al. Search for intracranial aneurysm susceptibility gene(s) using Finnish families. BMC Med Genet 2002;3:7.

7 Tanzi RE, Bertram L. New frontiers in Alzheimer's disease genetics. Neuron 2001;32:181-4.

8 MARS study group. Risks and benefits of screening for intracranial aneurysms in first-degree relatives of patients with sporadic subarachnoid hemorrhage. N Engl J Med 1999;341:1344-50.

9 Vermeulen M, Lindsay KW, Murray GD, et al. Antifibrinolytic treatment in subarachnoid hemorrhage. N Engl J Med 1984;311:432-7.

10 Chambers WR, Harper BF, Simpson JR. Familial incidence of congenital aneurysms of cerebral arteries. JAMA 1954;155:358-9.

11 Chakravorty B, Gleadhill CA. Familial incidence of cerebral aneurysms. BMJ 1966;5480:147-8.

12 Beumont PJV. The familial occurrence of berry aneurysm. J Neurol Neurosurg Psychiatry 1968;31:399-402.

13 Bannerman RM, Ingall GB, Graf CJ. The familial occurrence of intracranial aneurysms. Neurology 1970;20:283-92.

14 Kak V, Gleadhill C, Bailey IC. The familial incidence of intracranial aneurysms. J Neurol Neurosurg Psychiatry 1970;33:29-33.

15 Brisman R, Abbassioun K. Familial intracranial aneurysms. J Neurosurg 1971;34:678-82.

16 Bentzen N. Family incidence of intracranial aneurysms: case reports. NZ Med J 1972;75:153-5.

17 Edelsohn L, Caplan L, Rosenbaum AE. Familial aneurysms and infundibular widening. Neurology 1972;22:1056-60.

18 Nagae K, Goto I, Ueda K, et al. Familial occurrence of multiple intracranial aneurysms. Case report. J Neurosurg 1972;37:364-7.

19 Jain KK. Familial intracranial aneurysms. Acta Neurochir 1974;30: 129-37.

20 Acosta-Rua GJ. Familial incidence of ruptured intracranial aneurysms. Report of 12 cases. Arch Neurol 1978;35:675-7.

21 Fox JL, Ko JP. Familial intracranial aneurysms. Six cases among 13 siblings. J Neurosurg 1980;52:501-3.

22 Fox JL. Familial intracranial aneurysms. Case report. J Neurosurg 1982;57:416-17 
23 Morooka Y Waga S. Familial intracranial aneurysms: report of four families. Surg Neurol 1983;19:260-2.

24 Verdura J, Resnikoff S, Rosenthal J, et al. Familial intracranial aneurysms, with two occurring at the distal anterior cerebral artery. Neurosurgery 1983;12:214-16.

25 Maroun FB, Murray GP, Jacob JC, et al. Familial intracranial aneurysms: report of three families. Surg Neurol 1986;25:85-8.

26 Leblanc R, Lozano AM. Graves' disease and subarachnoid hemorrhage: a possible familial association. Can J Neurol Sci 1987;14:638-41.

27 Brodsky MC, Frenkel RE, Spoor TC. Familial intracranial aneurysm presenting as a subtle stable third nerve palsy. Case report. Arch Ophthalmol 1988;106:173.

28 Mellergard $P$, Ljunggren $B$, Brandt $L$, et al. HLA-typing in a family with six intracranial aneurysms. Br J Neurosurg 1989:3:479-85.

29 Elshunnar KS, Whittle IR. Familial intracranial aneurysms: report of five families. Br J Neurosurg 1990;4:181-6.

30 Bailey IC. Familial subarachnoid haemorrhage. Ulster Med J 1993;62:119-26.

31 Ronkainen A Hernesniemi J, Ryynanen M. Familial subarachnoid hemorrhage in east Finland, 1977-1990. Neurosurgery 1993;33 787-96.

32 Leblanc R, Melanson D, Tampieri D, et al. Familial cerebral aneurysms: a study of 13 families. Neurosurgery 1995;37:633-8.
33 Kaminogo M Yonekura M, Shibata S. Incidence and outcome of multiple intracranial aneurysms in a defined population. Stroke 2003 Jan;34:16-21.

34 Roos EJ, Rinkel GJE, Velthuis BK, et al. The relation between aneurysmal size and outcome in patients with subarachnoid hemorrhage. Neurology 2000;54:2334-6.

35 Penrose LS. The problem of anticipation in pedigrees of dystrophia myotonica Ann Eugenics 1948;14:125-32.

36 ACROSS study. Epidemiology of aneurysmal subarachnoid hemorrhage in Australia and New Zealand. Stroke 2000;31:1843-50.

37 Ashizawa T, Zoghbi H. Diseases with trinucleotide repeat expansions. In: Current neurology. Amsterdam: IOS Press, 1997:79-135

38 David G, Durr A, Stevanin G, et al. Molecular and clinical correlations in autosomal dominant cerebellar ataxia with progressive macular dystrophy (SCA7). Hum Mol Genet 1998;7:165-70.

39 Teunissen LL, Rinkel GJE, Algra A, et al. Risk factors for subarachnoid hemorrhage: a systematic review. Stroke 1996;27:544-9.

40 Rinkel GJE, Diibuti $M$, Algra A, et al. Prevalence and risk of rupture of intracranial aneurysms. Stroke 1998;29:251-6.

41 Shinton R, Palsingh J, Williams B. Cerebral haemorrhage and berry aneurysm: evidence from a family for a pattern of autosoma dominant inheritance. J Neurol Neurosurg Psychiatry $1991 ; 54: 838-40$ 